\title{
Probe-based Schemes to Guarantee Lightpath Quality of Transmission (QoT) in Transparent Optical Networks
}

\author{
N. Sambo (1), F. Cugini (2), I. Cerutti (1), L. Valcarenghi (1), P. Castoldi (1), \\ J. Poirrier (3), E. Le Rouzic (3), C. Pinart (4) \\ 1: Scuola Superiore Sant'Anna, Pisa, Italy, e-mail: nicola.sambo@sssup.it \\ 2: CNIT, Pisa, Italy \\ 3: France Telecom RD, Lannion, France \\ 4: CTTC, Centre Tecnologic de Telecomunicacions de Catalunya, Barcelona, Spain
}

\begin{abstract}
Two probe-based schemes are proposed to dynamically guarantee lightpath QoT in transparent optical networks. Low blocking and fast set up times are achieved within few lightpath set up attempts.
\end{abstract}

\section{Introduction}

In transparent dynamic optical networks, activation of lightpath for data transmission requires that lightpath Quality of Transmission (QoT) is ensured. In [1], GMPLS routing and signaling protocol extensions are proposed for estimating the lightpath QoT prior to lightpath set-up procedure. However, lightpath QoT estimation may be inaccurate.

In [2], an enhanced version of the routing-based scheme, namely Create-and-Wait (CW) scheme, is proposed. In CW, relevant QoT parameters are collected by the monitoring equipment and flooded by the routing protocol within hundreds of ms. Upon a lightpath request, an OSNR-based or Q-factor-based model estimates the expected lightpath QoT (e.g., estimated Bit Error Rate (BER)). When the estimated QoT meets the required QoT level, the lightpath set up procedure is triggered. The main feature of the $\mathrm{CW}$ scheme is the generation of probe traffic, to be transmitted on the lightpath prior to activation (i.e., data transmission). The QoT measurements (e.g., BER) taken on the probe traffic at the destination can validate the estimated QoT. If the measured QoT does not meet the required QoT level, the lightpath is torn down and a successive set up attempt is triggered on a different route.

When using CW scheme, acceptable QoT is always guaranteed for each activated lightpath. However, OSNR-based QOT models, that take into account multiple physical impairments, are complex and may need a large amount of QoT-related information. This may negatively impact the control plane stability and scalability. Moreover, OSNR-based model may require complex and expensive monitoring equipment. Furthermore, some physical impairments (e.g., non-linear impairments) are difficult to model and to relate to OSNR.

This paper proposes and evaluates two different schemes, based on probe traffic measurements. The proposed probe-based schemes reduce the $\mathrm{CW}$ scheme implementation complexity. The advertisement of QoT information and the models for QoT estimation are either simplified (by using an equivalent-length model) or avoided.
Proposed probe-based scheme implementations The first proposed scheme is referred to as Equivalent-Length Probe Scheme (EL-PS). In EL-PS, the Equivalent Length (EL, [3]) is the only QoT parameter. The EL value for each network link is typically static and advertised by the routing protocol with EL extensions [3]. In EL-PS, lightpath QoT is estimated by linearly combining the $E L$ values of the traversed links. If the total $E L$ is below a predefined Maximum EL $\left(\mathrm{M}_{\mathrm{EL}}\right)$ threshold, the lightpath set up is triggered and the probe traffic is transmitted over the established (not yet activated) lightpath.

The second proposed scheme is called Probe Scheme (PS). In PS, no QoT estimation is carried out, i.e., no need for routing protocol extensions. Lightpath set up procedure is triggered for each lightpath requests. Then, QoT is evaluated by means of the probe traffic measurements.

In both schemes, if the probe measurement meets the required QoT level, the lightpath is activated for data transmission. Otherwise, another set up attempt is triggered on a different route. Probe measurements are performed using the Link Management Protocol (LMP). In particular, the LMP Link Connectivity Verification procedure transmits a LMP Test message (i.e., a pre-defined sequence of bits), over the lightpath not yet activated. BER measurements taken at the destination node are reported to the source node in the LMP BER Estimate field defined in [4].

\section{Simulation Results}

The performance of the proposed EL-PS and PS schemes is evaluated by means of a custom C++ event-driven simulator. A Pan-European topology with 32 links and 17 nodes is considered [5]. Lightpath requests are generated following a Poisson process and uniformly distributed among all node pairs. Lightpath routes are randomly selected between the shortest paths in terms of number of traversed hops. Lightpath wavelength selection is first fit. The considered $E L$ value is the link length expressed in $\mathrm{km}$ while the EL threshold $\mathrm{M}_{\mathrm{EL}}$ is selected as the length that guarantees that lightpaths with acceptable QoT are never rejected. QOT measurement values 


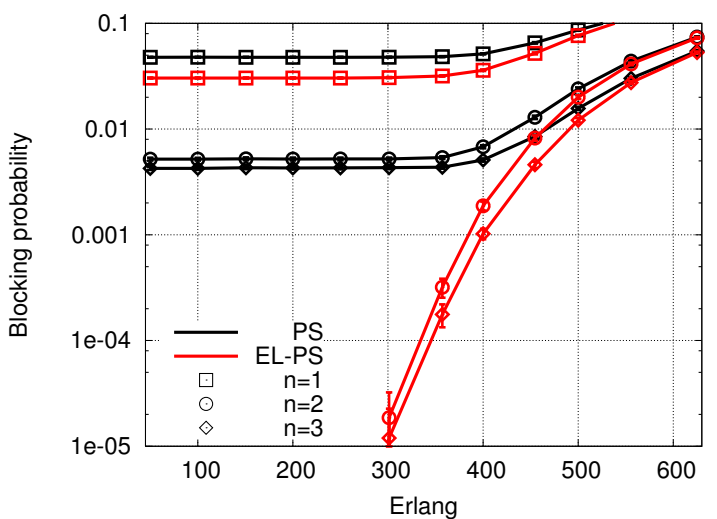

Fig 1: Network $N_{A}$ : Blocking probability at the $n$-th set up attempt.

are emulated considering the OSNR-based QoT model utilized in [5], taking into account Amplifier Spontaneous Emission (ASE), Polarization Mode Dispersion (PMD), chromatic dispersion, and selfphase modulation. Among the considered physical impairments, ASE and PMD are the most stringent for QoT. When QoT is not met or not enough resources are available in the network, lightpath set up is blocked and up to 2 additional set up attempts are triggered. Two different physical networks are considered for the Pan-European topology. The first one, $\mathrm{N}_{\mathrm{A}}$, is a realistic worst case scenario where a percentage $\left(\mathrm{P}_{A}=79 \%\right)$ of shortest routes traversing a number of hops equal to the network diameter $(D=5)$ is unable to meet QoT requirements. In the second one, $\mathrm{N}_{\mathrm{B}}$, the link impairments are less detrimental, i.e. $P_{B}=24 \%$. Two different equipment scenarios are considered, $S_{1}$ and $S_{2}$, which represent a lower and upper bound on expected equipment performance.

Table 1 summarizes the main lightpath set up operations including probe traffic generation and QoT measurement, in the two considered scenarios (i.e., $\mathrm{t}_{1}=500 \mathrm{~ms}$ in $\mathrm{S}_{1}$ and $\mathrm{t}_{2}=10 \mathrm{~s}$ in $\mathrm{S}_{2}$ ).

Fig. 1 and 2 show the lightpath blocking probability of EL-PS and PS schemes at the $n$-th set up attempt $(n \leq 3)$ in scenario $S_{2}$ versus the network load, for network $N_{A}$ and $N_{B}$, respectively. Fig. 1 shows that the EL-PS scheme outperforms the PS scheme especially after the first set up attempt. However, also the EL-PS scheme presents relevant blocking probability contributions especially at the first set up attempt. This is due to the $M_{E L}$ selection policy, which has the counter effect that some lightpaths with acceptable EL-estimated QoT may be rejected during the probe-based QoT measurement. Fig. 2 shows that the PS scheme is outperformed by the EL-PS scheme also in $\mathrm{N}_{\mathrm{B}}$. However, the performance gap between the two schemes is narrower and almost negligible after the first set up attempt. Moreover, results show that, by exploiting successive set up attempts, the overall blocking probability decreases. However, when $n$ increases from 2 to 3 only marginal reduction of blocking is achieved. This indicates that two set up attempts may be a good tradeoff between

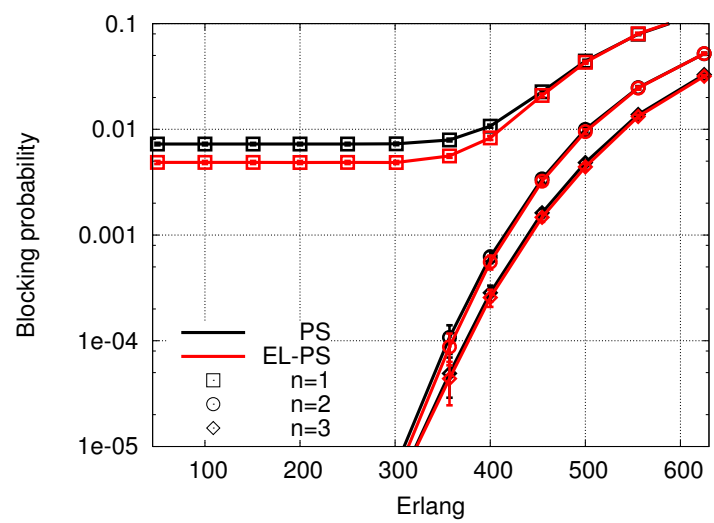

Fig 2: Network $N_{B}$ : Blocking probability at the $n$-th set up attempt.

performance and set up time. Similar blocking probability is also achieved by $S_{2}$ scenario (not included for space reason).

$S_{1}$ and $S_{2}$ scenarios differ in terms of the average time required to complete the lightpath set up (in Table 2, for network $N_{A}$ ). Results show that the EL-PS scheme performs better than the PS scheme in case of long lightpaths, i.e., traversing $D$ nodes. However, the overall average set up times are similar in $S_{1}$ and $\mathrm{S}_{2}$ scenarios.

Table 1: Lightpath set up operations

\begin{tabular}{|c|c|c|}
\hline & $\mathbf{S}_{1}[\mathrm{~ms}]$ & $\mathbf{S}_{2}$ [ms] \\
\hline Laser power \& frequency setting at Tx & 50 & 1000 \\
\hline Traversed node switching (e.g., WSS) & 45 & 1000 \\
\hline Propagation time (1000 km) & 5 & 5 \\
\hline Probe synchr. \& acquisition at Rx & 250 & 7000 \\
\hline Probe QoT measurement (e.g., BER) & 150 & 1000 \\
\hline Total & $\mathbf{5 0 0}$ & $\sim 10000$ \\
\hline
\end{tabular}

Table 2: Network $N_{A}$ : Average lightpath set up time

\begin{tabular}{|c|c|c|c|c|}
\hline \multirow{2}{*}{} & \multicolumn{2}{|c|}{$\mathbf{S}_{\mathbf{1}}\left(\mathbf{t}_{1}=\mathbf{5 0 0 m s}\right)[\mathbf{s}]$} & \multicolumn{2}{|c|}{$\mathbf{S}_{\mathbf{2}}\left(\mathbf{t}_{\mathbf{2}}=\mathbf{1 0} \mathbf{s}\right)[\mathbf{s}]$} \\
\cline { 2 - 5 } & All & D hops & All & D hops \\
\hline EL-PS & 10.3 & 10.3 & 0.53 & 0.54 \\
\hline PS & 10.4 & 11.4 & 0.54 & 0.60 \\
\hline
\end{tabular}

\section{Conclusions}

In this paper, two lightpath set up schemes based on probe traffic measurements, with or without a priori QoT estimation, are proposed for guaranteeing the required lightpath QoT in GMPLS transparent networks. Results show that the proposed PS achieves good performance without requiring protocol extensions for QoT, especially in transparent networks with few QoT impaired routes.

Acknowledgements: This work was supported by the BONE project, a Network of Excellence funded by the European Commission.

\section{References}

1. R. Martinez et al., Com Mag, Vol. 44, Dec. '06

2. Pinart C. et al., ICTON Conf., June ' 07

3. J. Strand, A. Chiu, RFC 4054, May '05

4. A. Fredette, J. Lang, RFC 4209, Oct '05

5. N. Sambo et al., ECOC Conf., Sept. '07 